\title{
Ultrasonic spectrum analysis for in vivo characterization of tumor microstructural changes in the evaluation of tumor response to chemotherapy using diagnostic ultrasound
}

\author{
Chun-yi Lin ${ }^{2+}$, Long-hui Cao ${ }^{3+}$, Jian-wei Wang ${ }^{1}$, Wei Zheng ${ }^{1}$, Yao Chen ${ }^{1}$, Zi-zhen Feng ${ }^{4}$, An-hua Li ${ }^{1}$
} and Jian-hua Zhou ${ }^{1 *}$

\begin{abstract}
Background: There is a strong need for early assessment of tumor response to chemotherapy in order to avoid the adverse effects of unnecessary chemotherapy and to allow early transition to second-line therapy. The purpose of this study was to determine the feasibility of ultrasonic spectral analysis for the in vivo characterization of changes in tumor microstructure in the evaluation of tumor response to chemotherapy using diagnostic ultrasound.

Methods: Experiments were approved by the regional animal care committee. Twenty-four MCF-7 breast cancer bearing nude mice were treated with adriamycin or sterile saline administered by intraperitoneal injection.

Ultrasonic radio-frequency (RF) data was collected using a clinically available ultrasound scanner (6-MHz linear transducer). Linear regression parameters (spectral slope and midband-fit) regarding the calibrated power spectra from the RF signals were tested to monitor tumor response to treatment. The section equivalent to the ultrasound imaging plane was stained with hematoxylin and eosin to allow for assessment of the density of tumor cell nuclei.

Results: Treatment with adriamycin significantly reduced tumor growth in comparison with the control group $(p=0.003)$. Significant changes were observed in the ultrasonic parameters of the treated relative to the untreated tumors $(p<0.05)$. The spectral slope increased by $48.5 \%$, from $-10.66 \pm 2.96$ to $-5.49 \pm 2.69$; the midband-fit increased by $12.8 \%$, from $-57.10 \pm 7.68$ to $-49.81 \pm 5.40$. Treated tumors were associated with a significant decrease in the density of tumor cell nuclei as compared with control tumors $(p<0.001)$.

Conclusions: Ultrasonic spectral analysis can detect changes in tumor microstructure after chemotherapy, and this will be helpful in the early evaluation tumor response to chemotherapy.
\end{abstract}

Keywords: Adriamycin, Chemotherapy, Cancer, Ultrasonic spectrum analysis, Microstructure

\section{Background}

Tumor malignancy is one of the principal diseases that adversely affect human health and quality of life. More than half of all patients diagnosed with a malignant tumor will receive chemotherapy. At the present time, chemotherapy is still one of the most important cancer treatment methods. Early evaluation of tumor response

\footnotetext{
* Correspondence: zjh96421@hotmail.com

${ }^{\dagger}$ Equal contributors

'Department of Ultrasound, State Key Laboratory of Oncology in South China, Sun Yat-Sen University Cancer Center, Guangzhou 510060, P.R. China Full list of author information is available at the end of the article
}

to chemotherapy in patients with cancer may help to avoid unnecessary treatment and enable the use of alternative therapies. Currently in clinical oncology and experimental therapeutics, assessment of tumor treatment response to chemotherapy relies on evaluating changes in tumor growth rate or volume weeks to months after the conclusion of a therapeutic protocol. These changes typically occur weeks to months late in the course of therapy. Functional techniques such as positronemission tomography (PET), dynamic contrast-enhanced magnetic resonance imaging (MRI) and dynamic contrast-enhanced computed tomography (CT) have

\section{Biomed Central}


been investigated regarding the early assessment of tumor response to chemotherapy; this involves depiction of reductions in the metabolic activity or the perfusion of the tumor, and some of the results have been promising [1-3]. However, the use of such imaging modalities to monitor tumor responses to chemotherapy can be limited either by their cost or exposure of the patient to radiation.

The aim of cancer therapy is to kill tumors by inducing cell death that can be used as an indicator of tumor response to therapy [4]. Increased tumor cell death early during the course of treatment, in preclinical and clinical studies, has been shown to be a good prognostic indicator of outcome $[5,6]$. Currently, standard methods for detecting cell death are invasive and require tissue biopsy for histologic analysis. Diffusion-weighted MRI has been used clinically to measure the increase in the apparent diffusion coefficient of water, which is thought to be increased in responding tumors owing to a decrease in cell density $[7,8]$. However, assessment of tumor response to chemotherapy requires repeated examinations and the cost of MRI limits its clinical use.

Ultrasound is an attractive modality for the assessment of tumor response to therapy because of the ease with which it can be repeated without exposing the patient or animal to any risk of radiation. Ultrasound imaging systems are also relatively inexpensive and mobile, a particular benefit for animal studies. Ultrasonic spectrum analysis is used to extract information regarding tissue structures that is not conveyed in conventional B-mode imaging. Spectrum analysis of frequency-dependent backscattered radiofrequency (RF) data has been used to characterize tissue microstructures in the diagnosis of prostate cancer, ocular tumors, and cardiac abnormalities [9-11] and to differentiate benign lymph nodes from malignant lymph nodes [12]. It has been shown theoretically that the spectral parameters are related to tissue microstructural properties (e.g., effective acoustic scatterer size and concentration) [13]. Ultrasonic spectral analysis with a high frequency transducer has been used to detect microstructural changes induced by radiotherapy, photodynamic therapy and chemotherapy in tumor xenografts [14-17]. Recently, Sadeghi-Naini and colleagues used ultrasonic spectral analysis with a Sonix RP system at an approximate center frequency of $7 \mathrm{MHz}$ to examine response to neoadjuvant chemotherapeutic in breast cancer patients and the preliminary results were promising [18]. However, this study was limited by the small number of cases $(n=10)$ and no confirmation of the changes in spectral parameters was obtained from evaluation of the corresponding tissue microstructures. The purpose of the present study was to determine the feasibility of ultrasonic spectral analysis for the in vivo characterization of tumor microstructural changes in the evaluation of tumor response to chemotherapy using diagnostic ultrasound.

\section{Methods}

\section{Animal model}

This study was approved by the Committee on the Ethics of Animal Experiments of the Sun Yat-Sen University under the guidelines of the National Institutes of Health for the care of laboratory animals. Human breast cancer cell line MCF-7 was obtained from State Key Laboratory of Oncology in Southern China. MCF-7 cells were grown in DMEN culture medium (Hyclone Co., UT, USA) supplemented with $10 \%$ fetal bovine serum (Gibco, Grand Island, NY, USA), penicillin $(50 \mathrm{U} / \mathrm{ml})$, and streptomycin $(50 \mu \mathrm{g} / \mathrm{ml})$ at $37^{\circ} \mathrm{C}$ in a humidified $5 \% \mathrm{CO}_{2}$ atmosphere. For inoculation, approximately $4 \times 10^{8} \mathrm{MCF}-$ 7 cells suspended in phosphate-buffered saline were injected subcutaneously into the right axillary fossa of 24 5- to 6-week old BALB/c nude female mice.

Twenty-four nude mice were randomly divided into two groups. Twelve of the nude mice were treated with adriamycin (Shenzhen Main Luck Pharmaceuticals Inc., Guangdong, China) diluted in sterile saline and administered once daily by intraperitoneal injection $(4 \mathrm{mg} / \mathrm{kg})$. Drug administration began at day 10 post tumor cell implantation when tumors had reached a size of $5 \mathrm{~mm}$. The remaining twelve nude mice in the control group were given the vehicle control medium (sterile saline) according the timing and dosing schedule used for the treated group.

\section{Ultrasound data acquisition}

Ultrasound imaging was performed at day 18 (i.e. 7 days after initiation of therapy). For the ultrasound imaging studies, each mouse was anesthetized by intraperitoneal injection of pentobarbital sodium at a dose of $75 \mathrm{mg} / \mathrm{kg}$ (Sigma, St. Louis, MO, USA). Centrifuged gel was used to minimize bubble formation in the gel and a stand-off gel pad was placed on the skin for scanning. A commercially available clinical ultrasound scanner, Sonix TOUCH (Ultrasonix Medical Corporation, Richmond, Canada) with a $6-\mathrm{MHz}$ linear transducer was used to simultaneously collect B-mode images and RF data from the treated and control tumors. For data acquisition, the ultrasound transducer was positioned such that the focal zone was at the same depth in each imaged specimen to control for any potential attenuation. All RF data were sampled with 16 bit resolution at a frequency of $35 \mathrm{MHz}$. All images and radiofrequency data were digitally recorded. All ultrasound examinations were performed by one radiologist, who was blinded to the treatment status. The greatest longitudinal, transverse and anteroposterior dimensions of tumors were measured in fundamental grayscale imaging 
using calipers. Tumor volume was calculated using the formula for a prolate ellipsoid: volume $=\pi / 6 \times$ length $\times$ width $\times$ depth. The largest cross-section plane of the tumor was imaged with the transducer held manually in this position throughout the examination. To evaluate the echogenicity changes in the tumor after chemotherapy, Adobe Photoshop 6.0 (Adobe Systems, San Jose, CA, USA) was used to measure the gray scale intensity of the ultrasound images.

\section{Ultrasonic spectral analysis}

The ultrasound RF data from was imported into MATLAB-based (v. 2009a: MathWorks, Natick, MA, USA) software developed in our lab for ultrasound spectral analysis. For the tumor, rectangular regions of interest (ROI) were centered approximately at the focal depth of the transducer. Three representative ROIs were selected for each tumor sample and averaged for the final analysis. RF data from each line segment were multiplied by a Hamming weighting function to suppress spectral lobes and the Fourier transform was computed.

The power spectrum was obtained by averaging the results from the independent scan lines. This power spectrum was divided by the power spectrum of the echo from a calibration target. A quartz flat was used as the calibration target and the perpendicular reflection off the quartz flat located at the focal point of the transducer was used to derive the power spectrum. Linear regression analysis was applied to the calibrated spectral amplitude to provide a best-fit line (Figure 1) [19]. To assess the role of ultrasonic spectrum analysis in monitoring tumor response to chemotherapy, two spectral parameters from the regression analysis were computed: the spectral slope (SS) and the midband fit (MBF). The $\mathrm{SS}$ is the slope of the linear regression of the calibrated spectrogram and the MBF is the value of the regression fit at the center frequency over which the spectrum was measured. The spectral parameters can potentially be mathematically related to the physical characteristics of ultrasound scatterers, such as the size and concentration of the ultrasound scatterers [20]. Further details on the theoretical and signal analysis considerations and the relationship between spectral parameters and tissue microstructure can be found elsewhere $[19,21]$.

\section{Histopathologic examination}

At the end of the experiment, mice were sacrificed using the standard method. Tumors were removed and fixed in $10 \%$ buffered formalin before paraffin processing. The tumor specimens were sectioned $(5 \mu \mathrm{m})$ at the largest cross sections corresponding to the ultrasound imaging planes. Sections were stained with hemotoxylin and eosin (H\&E) and assessed microscopically for changes in cell morphology.

Regions with the highest density of tumor cell nuclei were located by scanning the tissue sections under a $40 \mathrm{x}$ power microscope. After identification of the regions of highest density, ten different fields were randomly chosen within these regions at $400 x$ power. The $400 x$ histology images were then analyzed to measure the density of tumor cell nuclei by counting the number of nuclei in each image using Image Pro Plus software (Image Pro Plus 6.0, Media Cybernetics, Silver Spring, MD, USA) (Figure 2). The H\&E stained image segmentation was based on the following Hue-Saturation-Intensity (HSI) parameters: Hue (0-255), Saturation (0-255) and Intensity (0-120). The segmented areas in the images were filtered to count blue nuclei. This filtering used thresholds as follows: area (minimum $=50$ pixels) and box $\mathrm{x} / \mathrm{y}$ (minimum $=0.5$; maximum $=2$ ). The "split objects" function was used to separate cells touching each other. The average count of ten 400x images was used for the statistical analysis.

\section{Statistical analysis}

All statistical analyses were performed using SPSS version 16.0 (SPSS, Inc, Chicago, IL, USA). The KolmogorovSmirnov test was applied for evaluation of normal distribution and the Levene test for evaluation of homogeneity
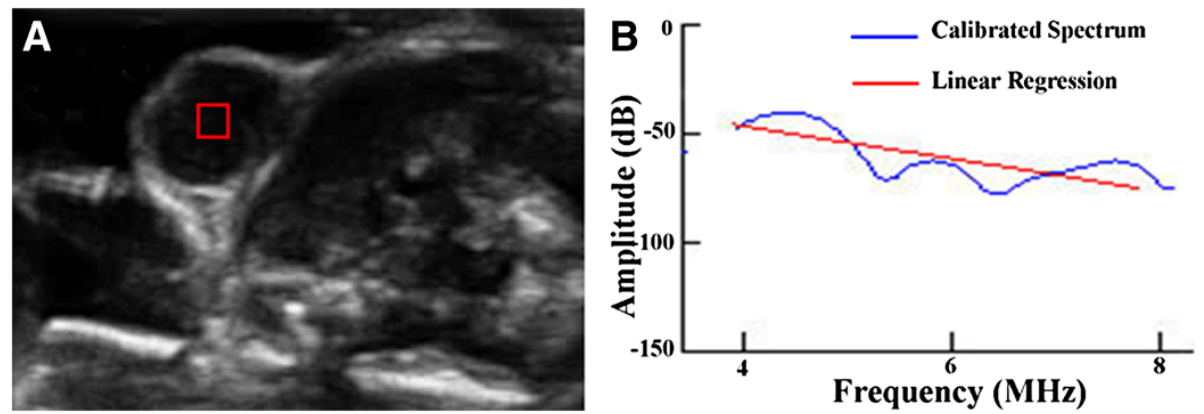

Figure 1 Ultrasound image and power spectrum. (A) Ultrasound image of the MCF7 tumor with the ROI located in the subcutaneous tumor tissue and (B) the corresponding calibrated power spectrum (blue curve) with linear regression (red line). 


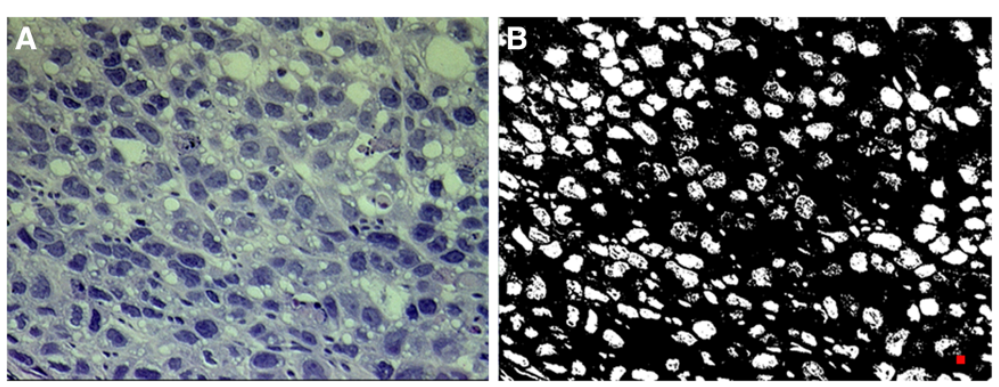

Figure 2 Histologic section and binary image. (A) A hemotoxylin and eosin (H\&E) stained image of the MCF-7 tumor and (B) the corresponding binary image indicating the presence of nuclei were identified using Image Pro Plus. The red square showed the area threshold used for counting the nuclei.

of variance. Student's $t$-test was used to determine the significant differences in measurement data between the treated and control tumors. A leave-one-out crossvalidation method was used to test the ability of slope and midband-fit to distinguish treated tumors from control tumors. A $p$ value of 0.05 or less was considered as being statistically significant.

\section{Results}

\section{Effect of adriamycin on tumor growth}

After treatment for 7 days, the mean tumor volumes of the treated and control tumors were $0.08 \pm 0.03 \mathrm{~cm}^{3}$ and $0.17 \pm 0.08 \mathrm{~cm}^{3}$, respectively. Treatment with adriamycin ( $4 \mathrm{mg} / \mathrm{kg}$ once daily) significantly reduced tumor growth in comparison to the control group $(p=0.003)$.

\section{Changes in ultrasonic spectral parameters}

After treatment for 7 days, the mean gray scale intensity of the control and the treated tumors in conventional B-mode ultrasound images was $15.40 \pm 3.86$ units and $19.00 \pm 1.25$ units, respectively. Treatment with adriamycin $(4 \mathrm{mg} / \mathrm{kg}$ once daily) significantly increased the gray scale intensity of the conventional B-mode ultrasound images as compared with control tumors $(p=0.009)$. Spectrum analysis of frequency-dependent backscattered radiofrequency data showed the difference in spectral parameters between the treated and control tumors. The mean spectral slope of the control and treated tumors was $-10.66 \pm 2.96 \mathrm{~dB} / \mathrm{MHz}$ and $-5.49 \pm 2.69 \mathrm{~dB} / \mathrm{MHz}$, respectively. Seven days treatment with adriamycin ( $4 \mathrm{mg} / \mathrm{kg}$ once daily) significantly increased spectral slope (by $48.5 \%$ ) in comparison to the control group $(p<0.001)$. The mean MBF of the
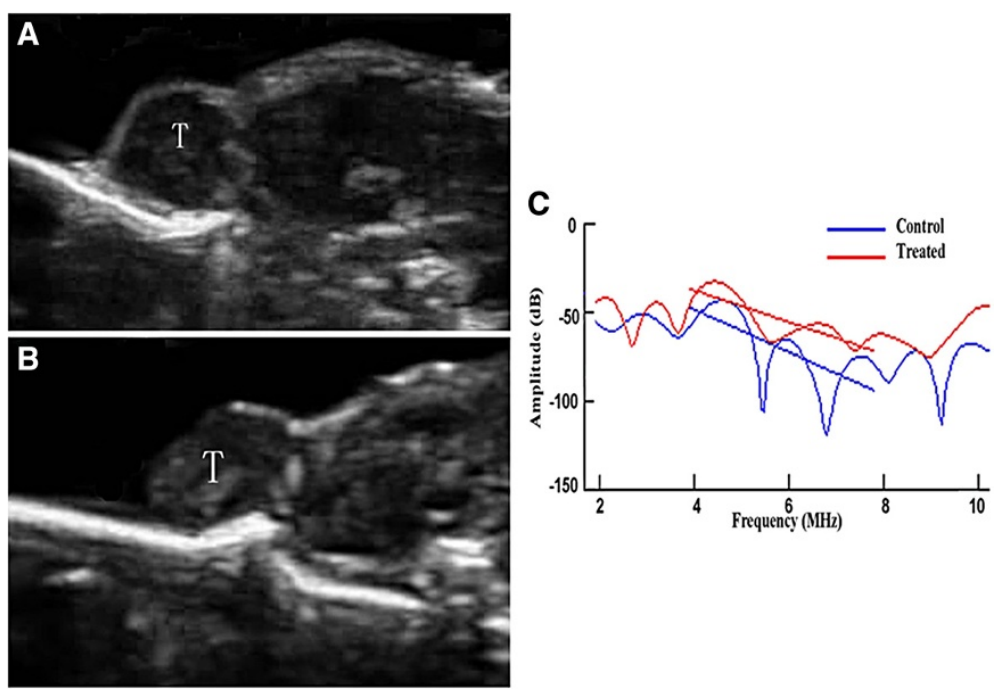

Figure 3 Representative ultrasound images and corresponding ultrasound spectral parameters. Ultrasound images for (A) a control tumor and (B) a treated tumor, and the corresponding ultrasound spectral parameter $(\mathbf{C})$ characterization of tumor responses to chemotherapy. The ultrasound images showed a noticeably brighter grayscale intensity in treated tumors (B) relative to control tumors (A). (C) Ultrasonic spectral analysis indicated a separation of the regression line between the control and the treated tumors. 
control and treated tumors was $-57.10 \pm 7.68$ and $-49.81 \pm$ $5.40 \mathrm{~dB}$, respectively. MBF was significantly increased (by $12.8 \%)$ after 7 days when the treatment tumor group was compared with the control group $(p=0.013)$ (Figure 3). In distinguishing between treated and control tumors using "leave-one-out" cross-validation, the correctly classified rate of slope and MBF was $87.5 \%$, and the sensitivity and specificity were $83.3 \%$ and $91.7 \%$, respectively.

\section{Histological changes}

The most prominent histological changes after chemotherapy in the treated tumors were related to the density of tumor cell nuclei, nuclear size and the extent of cytoplasmic and nuclear vacuolation of the tumor cells. The number of tumor cell nuclei evaluated in histological slice from treated tumors was $78.51 \pm 13.11$ counts per high-power field (HPF) and in control tumors was $334.50 \pm 44.57$ counts per HPF. Treatment with adriamycin (4 mg/kg once daily) significantly reduced the density of tumor cell nuclei in comparison to the control group $(p<0.001)$. H\&E staining revealed other microstructural change in treated tumors, involving nuclear structure manipulation (condensation and fragmentation) (Figures 4 and 5).

\section{Correlation between ultrasonic spectral parameters and histological results}

The density of cell nuclei density was found to be negatively correlated with spectral slope $(\mathrm{r}=-0.670, p<0.001)$ and MBF $(\mathrm{r}=-0.450, p=0.027)$.

\section{Discussion}

Reduction in tumor size is a late sign of effective chemotherapy, early response to chemotherapy is difficult to assess using conventional radiographic modalities [22]. Currently many cancer treatment regimens require several courses of chemotherapy before it can be determined whether or not the treatment has been effective. The availability of non-invasive methods for predicting and/or detecting therapeutic response to chemotherapy at an early stage of treatment would facilitate the rational design and individualization of therapy protocols for cancer patients and allow early transition to secondline therapy. The present study was undertaken to assess the usefulness of ultrasonic spectral analysis in the evaluation of conventional chemotherapy in a murine breast cancer model using tumor volume and the density of tumor cell nuclei as gold standards. This study has shown for the first time, using a clinically available 6

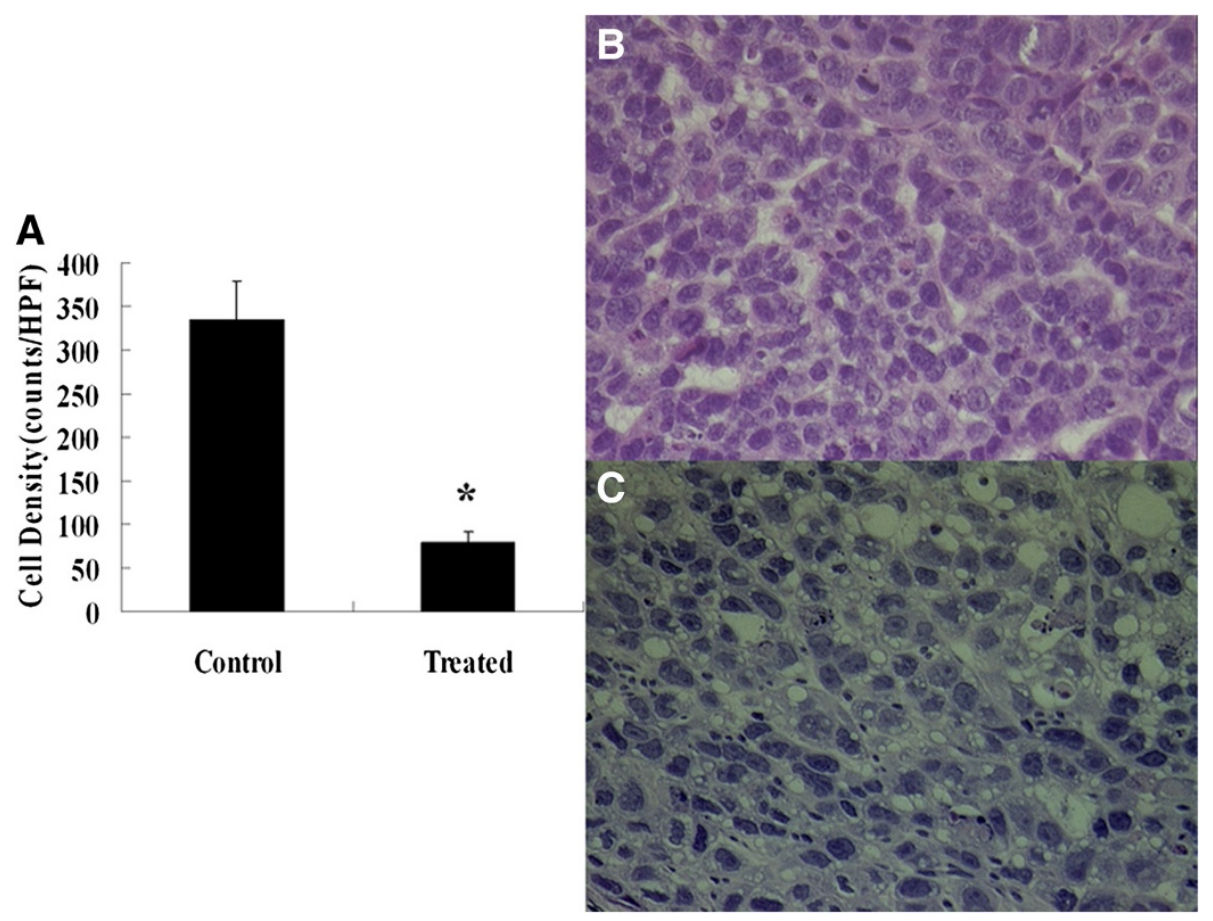

Figure 4 Histopathologic analysis of tumor cell nuclei density. (A) The graph displays the mean density of tumor cell nuclei in the control and treated tumors $\left({ }^{*}=p<.001\right)$. (B) and $(\mathbf{C})$ Representative photomicrographs of hemotoxylin and eosin stained sections of the control (B) and treated $(\mathbf{C})$ tumors. Staining revealed microstructural changes in the tumor treated with adriamycin, including a decreased density of cell nuclei, and cytoplasmic and nuclear vacuolation, and clumping of nuclear chromatin (original magnification, $\times 400$ ). 


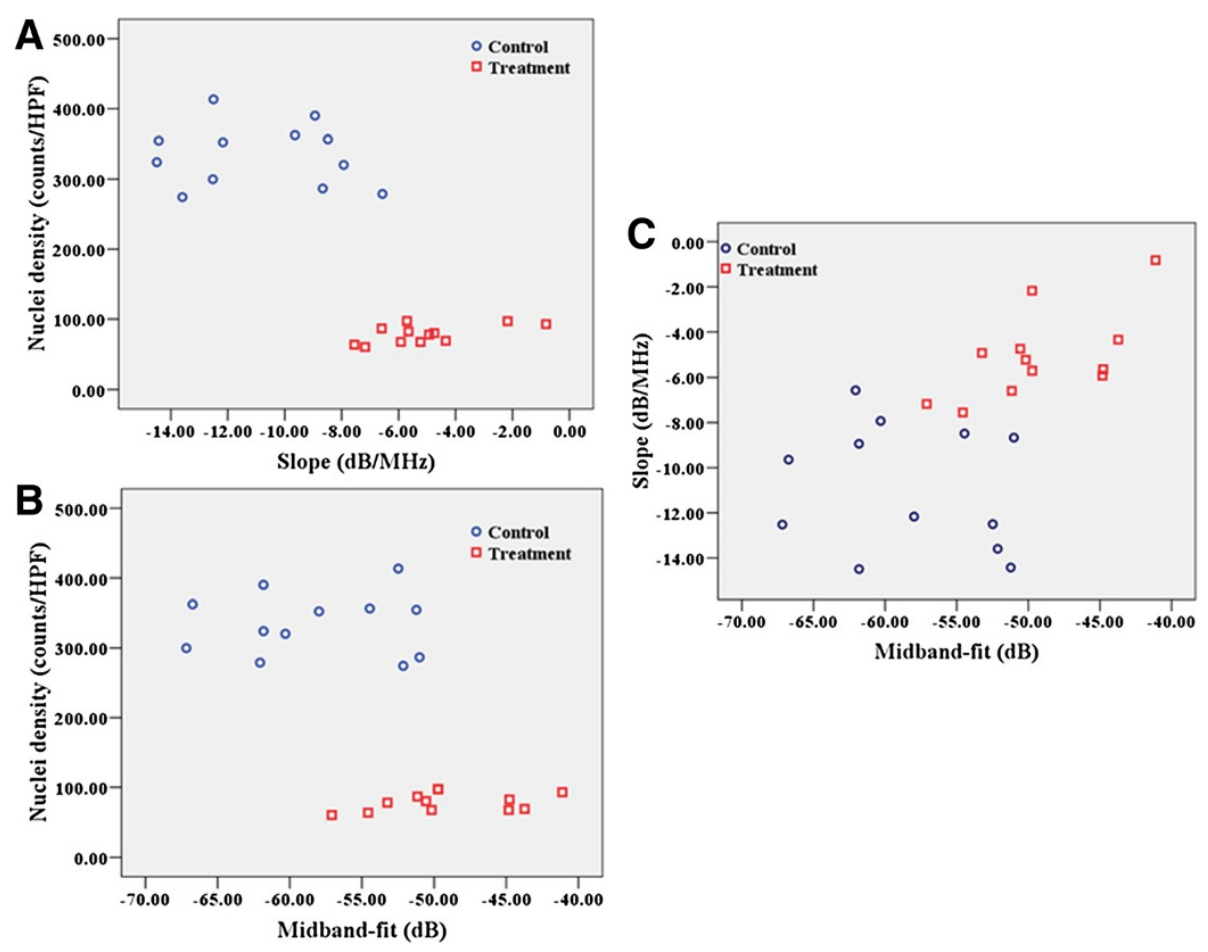

Figure 5 Scatter plots of changes in spectral parameters. (A) Spectral slope, (B) midband-fit versus cell nuclei density and (C) spectral slope versus midband-fit.

$\mathrm{MHz}$ ultrasound transducer, that chemotherapy effects can be characterized by means of ultrasonic spectral analysis in preclinical mouse cancer models. The changes in spectral parameters were interpreted as occurring as a direct consequence of cell death and changes in the density of cell nuclei after chemotherapy. This method was able to detect changes in the solid tumor microstructure after chemotherapy and, consequently has the potential to detect tumors that are responsive to treatment earlier than using conventional methods.

In the current study, histologic analysis revealed that the most prominent microstructural changes after chemotherapy were related to the density of cell nuclei, cytoplasmic and nuclear vacuolation and clumping of nuclear chromatin as indicated by the H\&E staining; these changes are frequently seen in breast cancer following neoadjuvant chemotherapy $[23,24]$. Our histologic observations suggested that the main changes in the ultrasonic spectral parameters after chemotherapy were related to microstructural changes regarding both the density of cell nuclei and cytomorphologic changes.

It has long been suggested that ultrasound backscatter variables may relate to the physical properties of tissues [20]. Ultrasonic spectral analysis techniques have been used by many investigators to add information regarding tissue microstructure to images generated by conventional ultrasound [25]. The spectral slope is an indicator of effective scatterer shape and size and an increase in spectral slope corresponds to a decrease in effective scatterer size [26]. The observed changes in the spectral slope after chemotherapy could be suggestive of structural changes in the tumor cells after treatment, namely cell shrinkage, nuclear condensation and fragmentation. The MBF is another measure of ultrasound backscatter and depends on multiple factors, including scatterer shape, size, concentration, and the acoustic impedance change between the acoustic scatterer and the surrounding medium [26]. Increase in MBF was seen in treated tumors after chemotherapy relative to control tumors. The mechanism behind this increase was broadly linked to changes in cell and nuclear morphology observed histologically after cell death. The strength of ultrasound backscatter depends both on scatterer size and differences in the mechanical properties of the scatterer, the surroundings (compressibility and density) and the scatterer number density (e.g., how many scatterers there are per unit volume) [15]. The changes in cell and nuclear sizes during the sequence of cell death resulted in an increase in the variance of cell sizes; a previous study by Vlad et al. [27] demonstrated that an increase in cellular size variance contributes to the increase in ultrasound backscatter during cell death. The decrease in the density of tumor cell nuclei and extensive changes in cytoplasmic and nuclear vacuolation of the 
tumor cells might result in changes in acoustic impedance, contributing to the increase in MBF.

Ultrasonic parameters, including spectral slope and MBF, have previously been used to characterize diseased tissue or tissue and cell samples exposed to different therapeutic agents [14-17,27,28]. Kolios and colleagues used ultrasonic spectral analysis to measure changes in the spectral slope and MBF in vitro for cell samples exposed to chemotherapeutic drugs [29]. Significant increases in spectral slope and MBF in treated cells were observed after exposure to chemotherapy that were in close agreement with theoretical predictions. However, more complex histological changes were observed in in vivo in mouse breast cancer models after chemotherapy than in vitro in cell samples exposed to chemotherapeutic drugs. In our study, other than cell shrinkage and nuclear condensation and fragmentation, the most prominent microstructural changes observed after chemotherapy were related to the decrease in the density of tumor cell nuclei, and cytoplasmic and nuclear vacuolation as revealed using $\mathrm{H} \& \mathrm{E}$ staining. Moreover, high-frequency ultrasound has been used in previous studies to detect apoptosis in vivo in cell samples and animal systems exposed to different anticancer therapies [14-17,29]. A penetration depth of 2-5 cm at frequencies of $10-30 \mathrm{MHz}$ limits the applicability of this technique to superficial regions. The present study confirmed that ultrasonic spectral analysis using a lower frequency of $6 \mathrm{MHz}$ could be used to monitor changes in tumor microstructure in a mouse breast cancer tumor model after chemotherapy. Sadeghi-Naini and colleagues used ultrasonic spectral analysis with a Sonix RP system at an approximate center frequency of $7 \mathrm{MHz}$ to examine neoadjuvant chemotherapeutic response in breast cancer patients, and their preliminary results were promising [18]. Lower frequency ultrasound penetrates much deeper, and previous studies have confirmed that the backscattered data from low frequency ultrasound could be potentially subjected to the same analysis to provide information regarding structural changes in the tissue at the cellular level [30-33].

Increased tumor cell death occurring early during the course of treatment, in both preclinical and clinical studies, has been shown to be a good prognostic indicator of outcome $[5,6]$. As a result of tumor cell death, the density of tumor cell nuclei will decrease significantly after chemotherapy [23,24,34]; this has become a marker for the detection of early indications of tumor response to chemotherapy [4]. Noninvasive diffusion-weighted MRI is a well studied imaging technique for quantifying the increase in the apparent diffusion coefficient of water caused by a decrease in tumor cell density within $2-4$ days, prior to visible changes in tumor morphology or size in patients with breast [35], brain [36], and ovarian [8] cancers that responded to treatment. However, the cost of diffusionweighted MRI limits its extensive use in monitoring tumor response to cancer therapies. In contrast, in the measurement of changes in water diffusion caused by cell death, ultrasonic spectral analysis provides a simple imaging approach that can directly characterize tumor microstructural changes (decreased density of tumor cell nuclei) after chemotherapy.

A potential limitation of our technique was that there were potential variations in matching the ultrasound image planes with the histological slices, due to differences in slice thickness between the ultrasound scanner and histological sections. In order to make ultrasound data correspond more closely to histologic measurement, the largest cross-section planes were used in both techniques. Another limitation was that there was no compensation for attenuation in our study, However, because of the fact that the tumors were close to the skin surface, and the center of the ROI was located at approximately $0.2 \mathrm{~cm}$ below the skin surface, attenuation compensation will not likely lead to significant changes in the results for a $6 \mathrm{MHz}$ transducer. Moreover, the limiting scatterer size for a $6-\mathrm{MHz}$ linear transducer will be about $85 \mu \mathrm{m}$ (13), which is much larger than the nuclear diameter (about $15 \mu \mathrm{m}$ ); consequently, it was difficult to identify the histological texture of the scatterer for the $6-\mathrm{MHz}$ transducer, and therefore to specify the histological changes that caused the changes in the spectral parameters.

\section{Conclusions}

In conclusion, this study indicated that ultrasonic spectral analysis provided a simple way to characterize tumor microstructural changes after chemotherapy using a clinically available $6 \mathrm{MHz}$ ultrasound transducer. A significant increase in spectral slope and MBF were detected using this noninvasive imaging technique after chemotherapy. This would allow tumor imaging before and at multiple times during treatment without the need for injecting specialized contrast agents as is required using other techniques (e.g. PET, dynamic contrastenhanced CT and dynamic contrast-enhanced MRI). This noninvasive technique shows considerable potential in the early assessment of tumor response to chemotherapy.

\section{Competing interests}

The authors declare that there is no conflict of interest that could influence the impartiality of the research reported.

\section{Authors' contributions}

$J H Z$ conceived the study; CYL, JWW, LHC, WZ, YC, AHL and JHZ performed the experiments, CYL, JWW, LHC and JHZ contributed to data analysis; LHC and $\mathrm{JHZ}$ wrote the paper. All authors read and approved the final

manuscript. 


\section{Acknowledgments}

This work was supported by National Natural Science Foundation of China (No. 81271578), the Fundamental Research Funds for the Central Universities (No. 09ykpy56), Scientific Research Foundation for the Returned Overseas Chinese Scholars, State Education Ministry and Funds for Pearl River Science \& Technology Star of Guangzhou City.

\section{Author details}

${ }^{1}$ Department of Ultrasound, State Key Laboratory of Oncology in South China, Sun Yat-Sen University Cancer Center, Guangzhou 510060, P.R. China. ${ }^{2}$ School of Electronic and Information Engineering, South China University of Technology, Guangzhou 510640, P.R. China. ${ }^{3}$ Department of Anesthesiology, State Key Laboratory of Oncology in South China, Sun Yat-Sen University Cancer Center, Guangzhou 510060, P.R. China. ${ }^{4}$ Department of Radiation Oncology, State Key Laboratory of Oncology in South China, Sun Yat-Sen University Cancer Center, Guangzhou 510060, P.R. China.

Received: 6 November 2012 Accepted: 14 June 2013 Published: 21 June 2013

\section{References}

1. Groheux D, Giacchetti S, Espié M, Rubello D, Moretti JL, Hindié E: Early monitoring of response to neoadjuvant chemotherapy in breast cancer with 18F-FDG PET/CT: defining a clinical aim. Eur J Nucl Med Mol Imaging 2011, 38:419-425.

2. Ah-See ML, Makris A, Taylor NJ, Harrison M, Richman PI, Burcombe RJ, Stirling JJ, D'Arcy JA, Collins DJ, Pittam MR, Ravichandran D, Padhani AR: Early changes in functional dynamic magnetic resonance imaging predict for pathologic response to neoadjuvant chemotherapy in primary breast cancer. Clin Cancer Res 2008, 14:6580-6589.

3. Bellomi M, Petralia G, Sonzogni A, Zampino MG, Rocca A: CT perfusion for the monitoring of neoadjuvant chemotherapy and radiation therapy in rectal carcinoma: initial experience. Radiology 2007, 244:486-493.

4. Brindle K: New approaches for imaging tumour responses to treatment. Nat Rev Cancer 2008, 8:94-107.

5. Ellis PA, Smith IE, McCarthy K, Detre S, Salter J, Dowsett M: Preoperative chemotherapy induces apoptosis in early breast cancer. Lancet 1997. 349:849.

6. Chang J, Ormerod M, Powles TJ, Allred DC, Ashley SE, Dowsett M: Apoptosis and proliferation as predictors of chemotherapy response in patients with breast carcinoma. Cancer 2000, 9:2145-2152.

7. Hamstra DA, Galbán CJ, Meyer CR, Johnson TD, Sundgren PC, Tsien C, Lawrence TS, Junck L, Ross DJ, Rehemtulla A, Ross BD, Chenevert TL: Functional diffusion map as an early imaging biomarker for high-grade glioma: correlation with conventional radiologic response and overall survival. J Clin Oncol 2008, 26:3387-3394.

8. Kyriazi S, Collins DJ, Messiou C, Pennert K, Davidson RL, Giles SL, Kaye SB, Desouza NM: Metastatic ovarian and primary peritoneal cancer: assessing chemotherapy response with diffusion-weighted MR imaging-value of histogram analysis of apparent diffusion coefficients. Radiology 2011, 261:182-192

9. Liu T, Mansukhani MM, Benson MC, Ennis R, Yoshida E, Schiff PB, Zhang P, Zhou J, Kutcher GJ: A feasibility study of novel ultrasonic tissue characterization for prostate-cancer diagnosis: 2D spectrum analysis of in vivo data with histology as gold standard. Med Phys 2009, 36:3504-3511.

10. Liu T, Lizzi FL, Silverman RH, Kutcher GJ: Ultrasonic tissue characterization using 2-D spectrum analysis and its application in ocular tumor diagnosis. Med Phys 2004, 31:1032-1039.

11. Yang M, Krueger TM, Miller JG, Holland MR: Characterization of anisotropic myocardial backscatter using spectral slope, intercept and midband fit parameters. Ultrason Imaging 2007, 29:122-134.

12. Kumon RE, Pollack MJ, Faulx AL, Olowe K, Faroog FT, Chen VK, Zhou Y, Wong RC, Isenberg GA, Sivak MV, Chak A, Deng CX: In vivo characterization of pancreatic and lymph node tissue by using EUS spectrum analysis: a validation study. Gastrointest Endosc 2010, 71:53-63.

13. Lizzi FL: Ultrasonic scatterer-property images of the eye and prostate. Proc 1997 IEEE Ultrasonics Symp 1997:1109-1116.

14. Vlad RM, Brand S, Giles A, Kolios MC, Czarnota GJ: Quantitative ultrasound characterization of responses to radiotherapy in cancer mouse models. Clin Cancer Res 2009, 15:2067-2075.
15. Banihashemi B, Vlad R, Debeljevic B, Giles A, Kolios MC, Czarnota GJ: Ultrasound imaging of apoptosis in tumor response: novel preclinical monitoring of photodynamic therapy effects. Cancer Res 2008, 68:8590-8596.

16. Lee J, Karshafian R, Papanicolau N, Giles A, Kolios MC, Czarnota GJ: Quantitative ultrasound for the monitoring of novel microbubble and ultrasound radiosensitization. Ultrasound Med Bio. 2012, 38:1212-1221.

17. Hwang JY, Park J, Kang BJ, Lubow DJ, Chu D, Farkas DL, Shung KK, Medina-Kauwe LK: Multimodality imaging in vivo for preclinical assessment of tumor-targeted doxorubicin nanoparticles. PLOS One 2012, 7:e34463.

18. Sadeghi-Naini A, Falou O, Czarnota GJ: Quantitative ultrasound spectral parametric maps: Early surrogates of cancer treatment response. In Prof. 34th Annual Intl. Conf. Proc. IEEE Eng Med Biol Soc. ; 2012:2672-2675.

19. Lizzi FL, Greenebaum M, Feleppa EJ, Elbaum M, Coleman DJ: Theoretical framework for spectrum analysis in ultrasonic tissue characterization. J Acoust Soc Am 1983, 73:1366-1373.

20. Lizzi FL, Astor M, Feleppa EJ, Shao M, Kalisz A: Statistical framework for ultrasonic spectral parameter imaging. Ultrasound Med Biol 1997, 23:1371-1382.

21. Lizzi FL, Astor M, Liu T, Deng C, Coleman DJ, Silverman RH: Ultrasonic spectrum analysis for tissue assays and therapy evaluation. Int J Imaging Syst Technol 1997, 8:3-10.

22. Hlatky $L$, Hahnfeldt $P$, Folkman J: Clinical application of antiangiogenic therapy: microvessel density, what it does and doesn't tell us. J Natl Cancer Inst 2002, 94:883-893.

23. Rajan R, Esteva FJ, Symmans WF: Pathologic changes in breast cancer following neoadjuvant chemotherapy: implications for the assessment of response. Clin Breast Cancer 2004, 5:235-238.

24. Rajan $R$, Poniecka A, Smith TL, Yang Y, Frye D, Pusztai L, Fiterman DJ, Gal-Gombos E, Whitman G, Rouzier R, Green M, Kuerer H, Buzdar AU, Hortobagyi GN, Symmans WF: Change in tumor cellularity of breast carcinoma after neoadjuvant chemotherapy as a variable in the pathologic assessment of response. Cancer 2004, 100:1365-1373.

25. Czarnota GJ, Kolios MC, Hunt JW, Sherar MD: Ultrasound imaging of apoptosis. DNA-damage effects visualized. Methods Mol Biol 2002, 203:257-277.

26. Lizzi FL, King DL, Rorke MC, Hui J, Ostromogilsky M, Yaremko MM, Feleppa EJ, Wai P: Comparison of theoretical scattering results and ultrasonic data from clinical liver examinations. Ultrasound Med Biol 1988, 14:377-385.

27. Vlad RM, Saha RK, Alajez NM, Ranieri S, Czarnota GJ, Kolios MC: An increase in cellular size variance contributes to the increase in ultrasound backscatter during cell death. Ultrasound Med Biol 2010, 36:1546-1558

28. Taggart LR, Baddour RE, Giles A, Czarnota GJ, Kolios MC: Ultrasonic characterization of whole cells and isolated nuclei. Ultrasound Med Biol 2007, 33:389-401.

29. Kolios MC, Czarnota GJ, Lee M, Hunt JW, Sherar MD: Ultrasonic spectral parameter characterization of apoptosis. Ultrasound Med Biol 2002, 28:589-597.

30. Oelze ML, Zachary JF, O'Brien WD Jr: Parametric imaging of rat mammary tumors in vivo for the purposes of tissue characterization. J Ultrasound Med 2002, 21:1201-1210.

31. Czarnota GJ, Papanicolau N, Lee J, Karshafian R, Giles A, Kolios MC: Novel low-frequency ultrasound detection of apoptosis in vitro and in vivo [abstract]. Ultrason Imaging 2008, 29:237-238.

32. Zhou J, Zhang P, Osterman KS, Woodhouse SA, Schiff PB, Yoshida EJ, Lu ZF, Pile-Spellman ER, Kutcher GJ, Liu T: Implementation and validation of an ultrasonic tissue characterization technique for quantitative assessment of normal-tissue toxicity in radiation therapy. Med Phys 2009, 36:1643-1650

33. Papanicolau N, Karshafian R, Sadeghian A, Kolios M, Czarnota G: Conventional frequency evaluation of tumor cell death in response to treatment in vivo (abstract). J Acoust Soc Am 2010, 128:2365.

34. Symmans WF, Peintinger $F$, Hatzis C, Rajan R, Kuerer H, Valero V, Assad L, Poniecka A, Hennessy B, Green M, Buzdar AU, Singletary SE, Hortobagyi GN, Pusztai $L$ : Measurement of residual breast cancer burden to predict survival after neoadjuvant chemotherapy. J Clin Oncol 2007, 25:4414-4422. 
35. Park SH, Moon WK, Cho N, Song IC, Chang JM, Park IA, Han W, Noh DY: Diffusion-weighted MR imaging: pretreatment prediction of response to neoadjuvant chemotherapy in patients with breast cancer. Radiology 2010, 257:56-63.

36. Chenevert TL, Stegman LD, Taylor JM, Robertson PL, Greenberg HS, Rehemtulla A, Ross BD: Diffusion magnetic resonance imaging: an early surrogate marker of therapeutic efficacy in brain tumors. J Natl Cancer Inst 2000, 92:2029-2036.

doi:10.1186/1471-2407-13-302

Cite this article as: Lin et al:: Ultrasonic spectrum analysis for in vivo characterization of tumor microstructural changes in the evaluation of tumor response to chemotherapy using diagnostic ultrasound. $B M C$ Cancer 2013 13:302.

\section{Submit your next manuscript to BioMed Central and take full advantage of:}

- Convenient online submission

- Thorough peer review

- No space constraints or color figure charges

- Immediate publication on acceptance

- Inclusion in PubMed, CAS, Scopus and Google Scholar

- Research which is freely available for redistribution 